\title{
Laparoscopic Manipulation of a Probe-based Confocal Laser Endomicroscope Using a Steerable Intravascular Catheter
}

\author{
Crispin Schneider, MD, * Adrien E. Desjardins, PhD, $\dagger$ \\ Kurinchi Gurusamy, PhD, ${ }^{*}$ David J. Hawkes, PhD, $\ddagger$ and Brian R. Davidson, MD*
}

\begin{abstract}
Probe-based confocal laser endomicroscopy is an emerging imaging modality that enables visualization of histologic details during endoscopy and surgery. A method of guiding the probe with millimeter accuracy is required to enable imaging in all regions of the abdomen accessed during laparoscopy. On the basis of a porcine model of laparoscopic liver resection, we report our experience of using a steerable intravascular catheter to guide a probe-based confocal laser endomicroscope.
\end{abstract}

Key Words: confocal laser endomicroscopy, laparoscopic liver resection, intravascular steerable catheter, intraoperative imaging

(Surg Laparosc Endosc Percutan Tech 2015;25:e83-e85)

C onfocal laser endomicroscopy (CLE) is increasingly used for in vivo microscopic assessment of tissues in experimental and clinical scenarios. CLE utilizes a miniaturized confocal laser microscope with a micrometer-scale resolution that allows for imaging of individual cells using exogenous or endogenous contrast agents. ${ }^{1}$

The 2 most common technical solutions used for performing CLE during laparoscopy or NOTES (natural orifice transluminal endoscopic surgery) are a bulkier rigid endomicroscope containing the required optics in its tip ${ }^{1}$ or a thin flexible optical fiber bundle design that conducts the excitation laser and transmits signals to an external laser scanning unit. ${ }^{2,3}$ Cellvizio (Mauna Kea Technologies, Paris, France) is a CE-marked CLE probe, which uses the latter fiber bundle, based design and comes with a diameter range of 0.3 to $4.5 \mathrm{~mm}$. This probe can be applied

Received for publication August 30, 2014; accepted January 22, 2015. From the $*$ Division of Surgery and Interventional Science; $†$ Department of Medical Physics; and \$Centre for Medical Image Computing, University College London, London, UK.

The experiments outlined in this article were funded by a Health Innovation Challenge Fund "Smart laparoscopic liver resection: integrated image guidance and tissue discrimination" by the Department of Health and the Wellcome Trust. B.R.D. is the principal investigator and A.E.D., K.G., and D.J.H. are all coapplicants on this grant.

This publication presents independent research commissioned by the Health Innovation Challenge Fund (HICF-T4-317), a parallel funding partnership between the Wellcome Trust and the Department of Health. The views expressed in this publication are those of the author(s) and not necessarily those of the Wellcome Trust or the Department of Health.

The authors declare no conflicts of interest.

Reprints: Crispin Schneider, MD, Division of Surgery and Interventional Science, 9th Floor, Royal Free Hospital, Rowland Hill Street, London NW32 PF, UK (e-mail: crispin.schneider.13@ ucl.ac.uk).

Copyright (C) 2015 Wolters Kluwer Health, Inc. All rights reserved. This is an open access article distributed under the Creative Commons Attribution License, which permits unrestricted use, distribution, and reproduction in any medium, provided the original work is properly cited. in a variety of situations including luminal endoscopy ${ }^{4-6}$ and laparoscopy. $2,7,8$

CLE could have major value for discrimination between normal and cancerous tissues during laparoscopic or open surgical procedures. Direct contact between probe tip and organ surface, ideally at a 90-degree angle, is important to produce optimal images. However, these fine fiber optic probes are expensive and easily damaged by laparoscopic instruments, which limit their use in the operative setting. Using standard laparoscopic instruments such as cholangiogram-catheter clamps or blunt laparoscopic graspers is likely to produce damage when grasping the fine probes directly by breaking the optical fibers contained within. We report a novel method of handling a probe-based CLE and its application during laparoscopic hemihepatectomy in a porcine model.

\section{MATERIALS AND METHODS}

Ethical approval from the local animal ethics committee was acquired before commencing experiments at Northwick Park Institute for Medical Research (London, UK). The technique was evaluated in 4 separate animals.

Domestic female mini-pigs with weights in the range of 60 to $80 \mathrm{~kg}$ were anesthetized and a pneumoperitoneum was established using the Veress technique. Laparoscopic $12 \mathrm{~mm}$ ports were placed subumbilically and superolateral to this in the right midclavicular line. Depending on surgeon's preference, 1 to two $5 \mathrm{~mm}$ ports were inserted 8 to $12 \mathrm{~cm}$ below the left subcostal margin with a further $5 \mathrm{~mm}$ port placed 10 to $15 \mathrm{~cm}$ under the right subcostal margin if required.

As part of laparoscopic imaging studies, liver necrosis was produced in the left lobe of the liver using microwave ablation under a previous general anesthetic. The method of steering the CLE probe was evaluated in this model while imaging the ablated areas and surrounding liver tissue. The 2 fluorescent contrast agents Fluorescein and Indocyanine green were injected intravenously 5 to 10 minutes before imaging to provide the required contrast. CLE imaging with the Cellvizio system was performed on the liver and other abdominal organs, using excitation wavelengths of $488 \mathrm{~nm}$ (blue light laser) and $660 \mathrm{~nm}$ (red light laser) with a probe size of $1.5 \mathrm{~mm}$ (Proflex-Z) and $0.94 \mathrm{~mm}$ (ProflexMiniZ), respectively. To manipulate the probe we inserted it into the lumen of a steerable intravascular catheter (Agilis; St Jude Medical, Saint Paul, MN) (Fig. 1) that can be introduced through a $5 \mathrm{~mm}$ laparoscopic port. This device is designed as a steerable introducer sheath for wirebased instruments (eg, guidewire, radiofrequency ablator) in interventional cardiac rhythm management. ${ }^{9,10}$ Agilis is available with 3 different degrees of curl (small, medium, and large) and an integrated valve prevents leakage of gas 


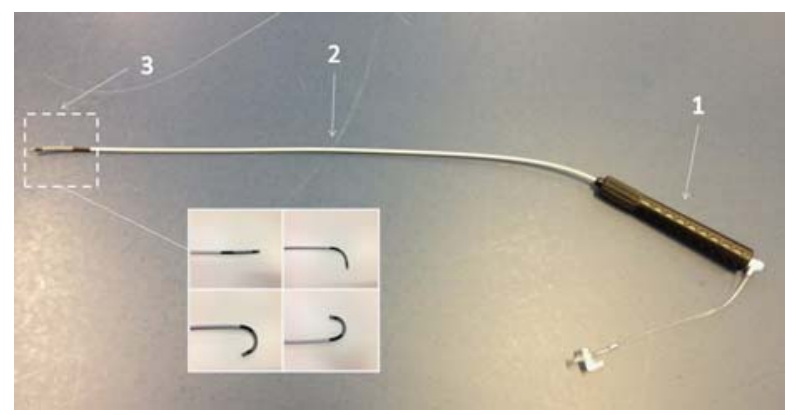

FIGURE 1. Full profile and possible degrees of tip flexion with the Agilis catheter. Full profile of Agilis catheter. (1) handle including controls, insertion port, and access port for flushing; (2) main body of catheter; (3) mobile tip that can be steered by the handle controls. The inset image shows possible degrees of tip flexion with the Agilis catheter that range from neutral to 180-degree retroflexion. The main body of the catheter (light gray) will slightly change shape when the tip is flexed extensively.

from the pneumoperitoneum. Controls in the handle allow the tip of the device to be flexed and retroflexed up to 180 degrees (Fig. 1), which, along with catheter rotation, enabled precise maneuvering and guidance of the tip even in a confined space (Fig. 2).

\section{RESULTS}

By twisting of the handle, the angle of the catheter tip can be subtly adjusted to allow optimal contact with the liver surface as shown in Figure 2. The blunt tip opening of the introducer did not provide any point of friction with the CLE probe and was atraumatic when in contact with hollow viscus and solid parenchymal organs. The probe is inserted through a gel port integrated in the handle of the
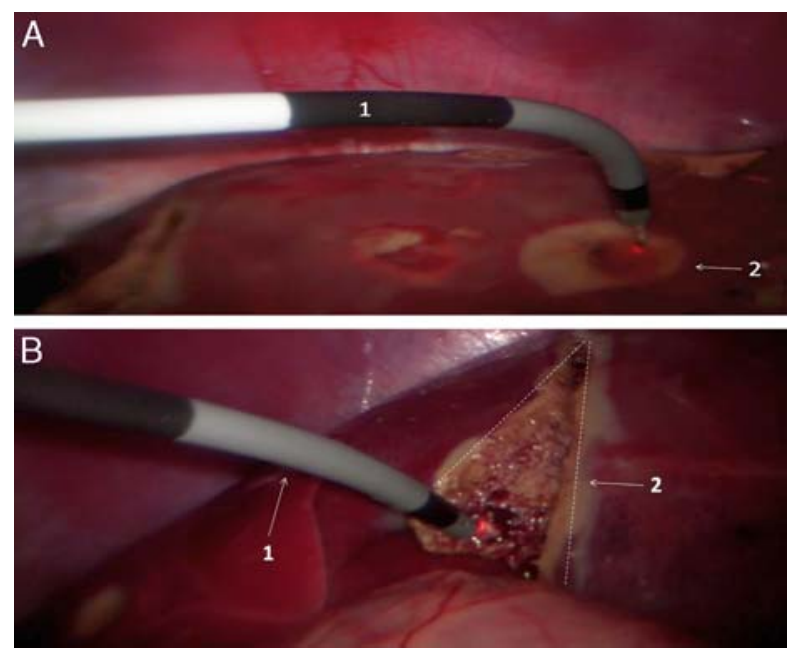

FIGURE 2. Handling of the catheter during laparoscopy. A, Catheter tip at 90-degree flexion during examination of the liver. (1) Agilis catheter; (2) ablated liver region simulating a "pseudotumour." B, Subtle control of the catheter tip enables CLE imaging in confined spaces. In this case the parenchymal transection plane is examined during left hemihepatectomy. (1) Agilis catheter; (2) liver transection plane. device that could readily accommodate probe sizes of up to $2 \mathrm{~mm}$.

Changing the probes longitudinal position in the port means that the Cellvizio tip could be advanced several centimeters from the catheter opening without affecting steering capabilities.

Because the catheters main body is durable and protects the confocal probe, it may be grasped directly to aid stability and maneuverability when required.

The smaller of the 2 probes (MiniZ) proved more difficult to manipulate because of the discrepancy between its circumference and the catheter tip aperture meant it was more mobile and unstable within the catheter lumen.

\section{DISCUSSION}

We described a novel method of handling a fiber optic-based CLE with a steerable intravascular catheter.

Although we have exclusively tested this approach for CLE, it may be useful for any emerging laparoscopic technologies that require accurate and subtle control of a long fiber or wire-based component that requires precise navigation on the surface of abdominal organs.

To improve probe handling, a method of securely fixing the length of the probe to the catheter, as moderate pressure on the probe tip leads to unwanted longitudinal movement of the probe within the catheter sheath, could be useful. One method could be to integrate an inflatable balloon within the handle of the device, which would narrow the lumen through which the probe traverses the handle and gently grip it to prevent longitudinal movement. A different method could be to fix a clamp to the proximal end of the handle that would grip the probe before it enters the gel port, which could be tightened and as needed to hold the probe in place. Cushioning the clamp with a soft material such as foam or gel would be essential to prevent damage or kinking of the probe.

So far, the method described in this paper was only tested in an animal model. It is a promising configuration to handle confocal probes in a clinical setting, especially as ethical approval to use 1-time-use steerable intravascular catheters in laparoscopic contexts could likely be readily obtained.

The Agilis system price of over $\$ 1000$ USD may be prohibitive for routine use, especially as it is designed and $\mathrm{CE}$ marked as a disposable device. It is unclear whether existing catheters could be sterilized sufficiently for reuse in laparoscopy, therefore Agilis should only be considered for single use at this stage. The findings reported in this article, however, may provide a platform from which manufacturers could develop a reusable, purpose-built device for laparoscopic CLE.

\section{REFERENCES}

1. Goetz M, Kiesslich R. Advances of endomicroscopy for gastrointestinal physiology and diseases. Am J Physiol Gastrointest Liver Physiol. 2010;298:G797-G806.

2. Von Delius S, Feussner H, Wilhelm D, et al. Transgastric in vivo histology in the peritoneal cavity using miniprobebased confocal fluorescence microscopy in an acute porcine model. Endoscopy. 2007;39:407-411.

3. Goetz M, Deris I, Vieth $M$, et al. Near-infrared confocal imaging during mini-laparoscopy: a novel rigid endomicroscope with increased imaging plane depth. J Hepatol. 2010;53: 84-90. 
4. Fuchs FS, Zirlik S, Hildner $\mathrm{K}$, et al. Confocal laser endomicroscopy for diagnosing lung cancer in vivo. Eur Respir J. 2013;41:1401-1408.

5. Giovannini M, Bories E, Monges G, et al. Results of a phase III study on intraductal confocal microscopy (IDCM) in patients with common bile duct (CBD) stenosis. Surg Endosc. 2011;25:2247-2253.

6. Kuiper $\mathrm{T}$, van den Broek FJ, van Eeden $\mathrm{S}$, et al. New classification for probe-based confocal laser endomicroscopy in the colon. Endoscopy. 2011;43:1076-1081.

7. Diana M, Dallemagne B, Chung H, et al. Probe-based confocal laser endomicroscopy and fluorescence-based enhanced reality for real-time assessment of intestinal microcirculation in a porcine model of sigmoid ischemia. Surg Endosc. 2014;28: 3224-3233.

8. Becker V, Wallace MB, Fockens P, et al. Needle-based confocal endomicroscopy for in vivo histology of intraabdominal organs: first results in a porcine model (with videos). Gastrointest Endosc. 2010;71:1260-1266.

9. Arya A, Hindricks G, Sommer P, et al. Long-term results and the predictors of outcome of catheter ablation of atrial fibrillation using steerable sheath catheter navigation after single procedure in 674 patients. Europace. 2010;12:173-180.

10. Rajappan K, Baker V, Richmond L, et al. A randomized trial to compare atrial fibrillation ablation using a steerable vs. a non-steerable sheath. Europace. 2009;11:571-575. 